Modern Physics Letters A

(C) World Scientific Publishing Company

\title{
Gravitational Waves and Perspectives for Quantum Gravity
}

\author{
Ilya L. Shapiro \\ Departamento de Física, ICE, Universidade Federal de Juiz de Fora, MG, Brazil \\ shapiro@fisica.ufjf.br \\ and \\ Tomsk State Pedagogical University and Tomsk State University, Tomsk, Russia
}

Ana M. Pelinson

Departamento de Física, CFM, Universidade Federal de Santa Catarina, SC, Brazil

ana.pelinson@gmail.com

Filipe de O. Salles

Departamento de Física, ICE, Universidade Federal de Juiz de Fora, MG, Brazil

fsalles@fisica.ufjf.br

Received (Day Month Year)

Revised (Day Month Year)

\begin{abstract}
Understanding the role of higher derivatives is probably one of the most relevant questions in quantum gravity theory. Already at the semiclassical level, when gravity is a classical background for quantum matter fields, the action of gravity should include fourth derivative terms to provide renormalizability in the vacuum sector. The same situation holds in the quantum theory of metric. At the same time, including the fourth derivative terms means the presence of massive ghosts, which are gauge-independent massive states with negative kinetic energy. At both classical and quantum level such ghosts violate stability and hence the theory becomes inconsistent. Several approaches to solve this contradiction were invented and we are proposing one more, which looks simpler than those what were considered before. We explore the dynamics of the gravitational waves on the background of classical solutions and give certain arguments that massive ghosts produce instability only when they are present as physical particles. At least on the cosmological background one can observe that if the initial frequency of the metric perturbations is much smaller than the mass of the ghost, no instabilities are present.
\end{abstract}

Keywords: Gravitational waves; Quantum gravity; Higher Derivatives.

PACS Nos.: 04.60.-m, 11.10.Jj, 04.30.Nk, 04.60.Bc,

\section{Introduction}

General relativity (GR) is a complete theory of classical gravitational phenomena, which proved valid at the wide range of energies and distances. However, as any other known physical theory, it has some limits of application. In order to establish 
these limits for GR, one has to review the most important solutions, which have specific important symmetries.

1) Spherically-symmetric solution is important for describing objects like planets, stars and black holes.

2) Isotropic and homogeneous metric is used to describe the zero-order approximation for the Universe.

It is well-known that both of these cases are characterized by singularities, that means the components of curvature tensor and energy density of matter become infinite in certain parts of the space-time manifolds. The natural interpretation is that GR is not valid at all scales and must be modified in the vicinity of the singularities. The problem is that, once the action of the theory and the corresponding equations of motion are modified, these changes can not be limited to the given regions of space-time. Indeed, certain modifications can become relevant and in some cases destructive in all points of the space-time manifolds. And this is the case for the modifications related to quantum effects of both matter fields and gravity itself.

One of the most natural reasons to modify GR is related to quantum effects. The expected scale of the quantum gravity (QG) effects is associated to the Planck units of length, time and mass. The three fundamental constants, namely speed of light $c$, Planck constant $\hbar$ and Newton constant $G$ can be used used uniquely to construct the universal Planck quantities (we set $c=1$ and $\hbar=1$ here)

$$
l_{P}=t_{P}=M_{P}^{-1}, \quad M_{P}=G^{-1 / 2} \approx 10^{19} \mathrm{GeV} .
$$

One may suppose that the fundamental Planck units indicate to the presence of a fundamental physics at the Planck scale $M_{P}$. Since $c, \hbar$ and $G$ are involved, it should be relativistic quantum and gravitational theory at the same time. Unfortunately, the dimensional approach does not tell us how this unification should happen. And, as we know, there are plenty of different ideas of what the relativistic quantum gravitational theory can look like. Now, in spite of a great variety of approaches, (more or less) all of them can be classified into three distinct general groups. One can:

(i) Quantize both gravity and matter fields. This is the most fundamental approach and the main subject of the present review, where we discuss only one particular (albeit very important) aspect of QG.

(ii) Quantize only matter fields on classical curved background (semiclassical approach). There are many good text-books and introduction reviews on the subject, let us just mention the books Refs. 1, 2, 3, 4, It is important that, different from quantum theory of metric itself, QFT and curved space-time are well-established notions, which passed many experimental and observational tests. If we put them together, we arrive at the new physics which is a decent object to explore - just because it describes some phenomena which certainly exist. On the other hand, there is no absolute certainty that metric itself should be quantized, since metric is, after all, different from all other fields.

(iii) Instead of quantizing gravity and/or matter, one can quantize something 
else. For example, in the case of (super)string theory both matter and gravity are induced. In this sense string theory is the most complete and consistent version of QG. However, there is still a fundamental question of why this theory should be regarded as quantum gravity. In other words, string theory is a very important approach to QG, but still it is one possible alternative among many possible approaches to QG.

An important detail is that all three possible lines of thought about QG have one common point, namely in all cases one meets higher derivatives in the effective action of gravity. In particular, the terms

$$
S_{H D}=\int d^{4} x \sqrt{-g}\left\{\alpha_{1} R_{\mu \nu \alpha \beta}^{2}+\alpha_{2} R_{\alpha \beta}^{2}+\alpha_{3} R^{2}+\alpha_{4} \square R\right\}
$$

actually emerge in all three approaches. In case of string theory, these higher derivative terms can be reduced and made non-offensive by means of the Zweibach reparametrization $\cdot^{[5}$ Indeed, one can perfectly well use the same approach in the first two cases, of (i) and (ii). It is sufficient to assume that, after the effective action of gravity (effective action of the external metric field, in case of (ii)) is found, one can perform the reparametrization of the metric

$$
g_{\mu \nu} \longrightarrow g_{\mu \nu}^{\prime}=g_{\mu \nu}+x_{1} R_{\mu \nu}+x_{2} R g_{\mu \nu}+\ldots,
$$

where the coefficients $x_{1,2, \ldots}$ are chosen in such a way that the higher derivative terms do not contribute to the propagator of gravitational perturbations. After that we define that the corresponding metric $g_{\mu \nu}^{\prime}$ is physical, exactly because in this parametrization there are no dangerous ghosts which can produce instabilities. In case of the fourth-derivative theory it is sufficient to require that the first two terms in (2), after the transformation (3), form the combination $\alpha_{1}\left(R_{\mu \nu \alpha \beta}^{2}-4 R_{\alpha \beta}^{2}\right)$, like in the Gauss-Bonnet topological term. In this case there is no gauge-independent tensor ghost in the spectrum. The coefficient of the last term, $R^{2}$, can not defined by the requirement of the absence of ghost, hence it represents an ambiguity. This is a particular case of the general situation, related to a serious ambiguity in the physical predictions of the gravitational theory based on the transformation (3) ${ }^{6}$

In the cases of (ii) and (iii) such a reparametrization concerns only external field (metric) and hence does not violate the unitarity of the $S$-matrix for quantum fields. As far as we know, the phenomenological consequences of this choice of the metric were never explored in the framework of the semiclassical approach (ii) and we will not deal with this problem in this review neither. Instead, we shall assume that no reparametrization like (3) is performed and discuss the effect of ghosts on the stability of the classical solutions.

In the next part of this short review we shall describe why higher derivatives are necessary in both semiclassical and quantum gravity. Furthermore, we present a brief review of ghosts and of the known approaches to avoid the inconsistencies related to them. Finally, we shall give a brief qualitative exposition of the results of our recent works ${ }^{7 / 8}$ and show that there are certain chances that the situation 
with ghosts is actually more optimistic when one is dealing with the physics of QG below the Planck scale.

The review is organized as follows. The next Section is devoted to the general status of higher derivatives in gravity. In Sect. 3 we consider the known methods of dealing with the ghost problem. Sect. 4 contains the main results on the effective approach to such ghosts and, finally, in Sect. 5 we draw our Conclusions.

\section{Why higher derivatives?}

Let us consider why higher derivatives are necessary in semiclassical and quantum gravity theories and what the ghost means.

\subsection{Semiclassical gravity}

The QFT in curved space requires introducing a generalized action of external gravity field. One can prove that the theory can be renormalizable only if such a vacuum action includes four derivative terms. We will not go into details, but just refer the reader to the books in Refs. 1, 3 for a general introduction, and to the recent paper in Ref. 9 for the most complete proof, including the case when non-covariant gauge fixing conditions are used. The necessary form of the "vacuum action" is as follows:

$$
S_{v a c}=S_{E H}+S_{H D}
$$

where

$$
S_{E H}=-\frac{1}{16 \pi G} \int d^{4} x \sqrt{-g}\{R+2 \Lambda\}
$$

is the Einstein-Hilbert action with the cosmological constant and the higher derivative term $S_{H D}$, defined in (2), can be recast in the most useful form as

$$
S_{H D}=\int d^{4} x \sqrt{-g}\left\{a_{1} C^{2}+a_{2} E+a_{3} \square R+a_{4} R^{2}\right\} .
$$

Here

$$
C^{2}=R_{\mu \nu \alpha \beta}^{2}-2 R_{\alpha \beta}^{2}+1 / 3 R^{2}
$$

is the square of the Weyl tensor and

$$
E=R_{\mu \nu \alpha \beta} R^{\mu \nu \alpha \beta}-4 R_{\alpha \beta} R^{\alpha \beta}+R^{2}
$$

is the integrand of the topological Gauss-Bonnet term.

In order to understand why the terms (4) are necessary, it is sufficient to consider the one-loop approximation, when the vacuum contribution reduce to the single bubble of matter field, as shown in Fig. 1 The external gravity can be implemented by means of the external tails of the field $h_{\mu \nu}$, where we assume the linear parametrization of the metric, $g_{\mu \nu}=\eta_{\mu \nu}+h_{\mu \nu}$. The simple bubble without tails of $h_{\mu \nu}$ has quartic divergences, the bubble with one vertex of matter-matter- $h_{\mu \nu}$ 


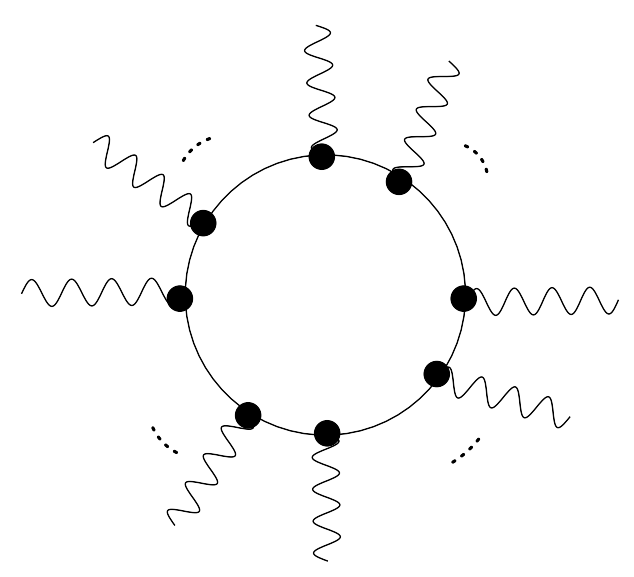

Fig. 1. General one-loop diagram of matter loop with gravitational external lines. The number of such lines coming to each vertex can be arbitrary. The divergences emerge only from the diagrams with one and two gravitational vertices. The power counting does not change at higher-loop order, if the matter theory is flat-space renormalizable.

interaction has quadratic divergences and, finally, the bubble with two such vertices has logarithmic divergences. Due to the general covariance of the counterterms, ${ }^{9}$ the possible logarithmically divergent structures are exactly those listed in (4).

In quantum gravity, higher derivative term like the square of the Weyl tensor indicate the presence of massive ghost, namely, a spin-two particle with negative kinetic energy. This leads to the problem with unitarity, at least at the tree level. But, in the semiclassical theory, gravity is external and unitarity of the gravitational $S$-matrix can be not requested. Therefore, the consistency conditions in this case can be relaxed to the following: (i) existence of physically reasonable solutions and (ii) their stability under small metric perturbations. Perhaps the most important point here is that the theory without the fourth-derivative terms (6) can not be consistent. If we do not include them into the classical action, these terms will emerge in the quantum corrections anyway, with infinite coefficients. The difference with the theory (44) would be that, without these terms in the classical action one can not control higher derivatives by means of multiplicative renormalization.

The last two important observations concerning higher derivatives are as follows. First, if we consider a more general theory, where metric is also an object of quantization, anyway one should definitely quantize both matter and gravity, for otherwise the QG theory would not be complete. Then the diagrams with matter internal lines in a complete QG are be exactly the same as in a semiclassical theory. This means one can not quantize metric without higher derivative terms in a consistent manner, since these terms are produced already within the semiclassical theory. The second point is that the effect of higher derivative terms can be Plancksuppressed in the classical solution, but this does not solve the problem, because the canonical wisdom tells us that more massive ghost would be even more destructive 
and lead to even faster explosion of the vacuum space-time (see, e.g., Ref. 10 for discussion). As we shall see in what follows, this is not necessary the case, so the huge mass of the ghost can provide a solution of the whole problem.

\section{2. (Super)renormalizable quantum gravity}

Let us now briefly consider the situation with higher derivatives in a proper QG. The standard traditional approach to QG assumes that the quantization can be performed using the variables

$$
\kappa h_{\mu \nu}=g_{\mu \nu}-\eta_{\mu \nu}
$$

It is assumed that this choice of the quantum metric is the "right" one. This choice enables one to deal with the well-defined object such as $S$ - matrix of the gravitational field $h_{\mu \nu}$. Let us note that in the case of quantum General Relativity the parametrization (9) corresponds to so-called Gaussian expansion of the action

$$
S_{E H}=-\frac{1}{\kappa^{2}} \int d^{4} x \sqrt{-g} R, \quad \kappa^{2}=16 \pi G .
$$

This means that in the variables (9) the second order (Gaussian approximation) of the action is $\kappa$-independent. In other words, in the absence of cosmological constant, the expansion in the coupling $\kappa$ and the flat-space expansion (9) are related notions. One of the consequences of this is that in the theories with a non-Gaussian fixed point $\kappa_{0}, \frac{11] 12}{12}$ the natural expansion is not the one in the powers of $\kappa$, but in the powers of $\kappa-\kappa_{0}$. In this case, the flat space is not a distinguished space-time from quantum viewpoint. On the other hand, this relation shows that the $S$-matrix approach to QG is relevant mainly in the standard perturbative QG. In the nonperturbative approaches, such as asymptotic safety scenarios, there may be no welldefined asymptotic states, no well-defined $S$-matrix and, hence, one should look for another criterion for the consistency of the theory, without necessary using the unitarity of the $S$-matrix as such a criterion. Once again, we arrive at the idea of checking stability of the physically relevant classical solutions in the theory. One can certainly consider this to be a minimal condition of consistency of the theory.

The construction of QG starts from some covariant action of gravity,

$$
S=\int d^{4} x \sqrt{-g} \mathcal{L}\left(g_{\mu \nu}\right),
$$

where $\mathcal{L}\left(g_{\mu \nu}\right)$ is the covariant Lagrangian density. The action can be (5), or (4), or some other, with more higher derivative terms. The gauge transformation is the diffeomorphism $x^{\prime \mu}=x^{\mu}+\xi^{\mu}$ and the metric transforms under it as

$$
\delta g_{\mu \nu}=g_{\mu \nu}^{\prime}(x)-g_{\mu \nu}(x)=-\nabla_{\mu} \xi_{\nu}-\nabla_{\nu} \xi_{\mu} .
$$

By using the method described in Ref. 13 for the case of (4), one can prove that the effective action of the metric, $\Gamma\left(g_{\mu \nu}\right)$ is also diffeomorphism invariant and the possible divergences are local, as usual in quantum field theory. Then one can use the notion of power counting to explore the possible form of these divergences. 
The universal formula for the superficial degree of divergence of a diagram with the power of momenta $r_{l}$ is the inverse of the propagator of some internal line, with the number of vertices $n$ and $K_{\nu}$ power of momenta in a given vertex, is

$$
D+d=\sum_{l_{\text {int }}}\left(4-r_{l}\right)-4 n+4+\sum_{\nu} K_{\nu} .
$$

Here $D$ is the superficial degree of divergence of a diagram and $d$ is the total number of derivatives on its external lines. Furthermore, one can use topological relations, e.g., the one between number of loops $p$, vertices $n$, and internal lines

$$
l_{\text {int }}=p+n-1 .
$$

As the first example, let us consider quantum GR, with the action (5). Obviously, the diagrams with the vertices $K_{\nu}=0$ will be less divergent. Therefore, for the sake of simplicity we consider only vertices with $K_{\nu}=2$. Taking $r_{l}=K_{\nu}=2$ and combining eqs. (13) with (14), we arrive at the estimate

$$
D+d=2+2 p .
$$

One can see that the diagrams with $D=0$ (that means only logarithmic divergences) require counterterms with the number of derivatives of the metric growing with the loop order according to $d=2+2 p$. This means that quantum GR is not renormalizable. This conclusion has been supported by direct calcu-

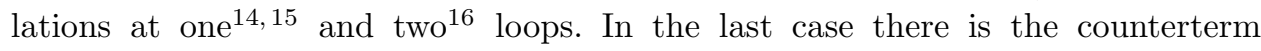
$R_{\mu \nu \alpha \beta} R_{\rho \sigma}^{\mu \nu} R^{\mu \nu \rho \sigma}$, which does not vanish on-shell, that means the divergences of the $S$-matrix of the theory can not be dealt with in a regular way. In the presence of matter the situation is similar even at the one-loop level.

Within the standard perturbative approach non-renormalizability means the theory has no predictive power. Every time we introduce a new type of counterterms, it is necessary to fix renormalization condition and this means performing a measurement. Since, according to (15), the number of counterterms is not restricted, before making a single prediction, in principle it is necessary to have an infinite amount of experimental data.

There are two possible way to the solutions of this problem:

1. Change standard perturbative approach to something else. This is the line of research which is beyond the framework of the present review. Let us only say that there are many options in this area, but their consistency and relation to the standard QG program are not clear, in all cases.

2. One can change to another theory as a starting point to construct QG.

The first option is widely explored in the asymptotic safety scenarios, in the effective approaches to QG and in the induced gravity paradigm (including string theory). Let us concentrate on the second choice, that means we will try to change the theory which is the subject of quantization. In this case one meets a great variety of different models. For example, the string theory belongs also to this class of approaches. However, if one is looking for a most simple and natural solution, 
the first option should be just to introduce higher derivatives into the gravitational action, starting from the terms (6). The reason is that we need these terms anyway for quantizing matter field. Since the full QG theory should include both metric and matter being quantized, one has to take care about the diagrams with the internal lines of matter fields and external ones of the gravitational field $h_{\mu \nu}$. But those diagrams are exactly the ones of the semiclassical theory and, as we already noted, they require the terms of both (6) and (5) to be included.

Now, if we do so, the situation in QG changes dramatically, because this new theory is renormalizable. The propagators and vertices in higher derivative QG (HDQG) are not the same as in quantum GR. In this case we have $r_{l}=4$ and there are vertices with $K_{4}, K_{2}$ and $K_{0}$. Then the superficial degree of divergence can be easily evaluated to give

$$
D+d=4-2 K_{2}-2 K_{0} .
$$

This theory is definitely renormalizable. Dimensions of counterterms, at all loops, are $4,2,0$, depending on the number of the vertices of the $K_{2}$ - and $K_{0}$-type.

Unfortunately, there is a high price to pay for renormalizability. The higher derivative QG based on the fourth-derivative action (4), possesses a massive spintwo gauge-independent excitation called massive ghost,

$$
G_{\text {spin }-2}(k) \sim \frac{1}{m^{2}}\left(\frac{1}{k^{2}}-\frac{1}{k^{2}+m^{2}}\right), \quad m \propto M_{P} .
$$

In the framework of linearized theory one can separate the massless and massive degrees of freedom. It is an easy exercise to check that the kinetic energy of the massive component is negative. For this reason this particle is called massive ghost. Indeed, the mass of this ghost is huge, of the Planck order of magnitude. The main point of this review is a new proposal concerning ghosts, which was originally done in Ref. 8 (see also Ref. 7).

Including even more derivatives was initially thought to move massive pole to a much higher mass scale. In Ref. 17 the following action was proposed

$$
\begin{aligned}
S & =S_{E H}+\int d^{4} x \sqrt{-g}\left\{a_{1} R_{\mu \nu \alpha \beta}^{2}+a_{2} R_{\mu \nu}^{2}+a_{3} R^{2}+\ldots\right. \\
& \left.+c_{1} R_{\mu \nu \alpha \beta} \square^{k} R^{\mu \nu \alpha \beta}+c_{2} R_{\mu \nu} \square^{k} R^{\mu \nu}+c_{3} R \square^{k} R+b_{1,2, . .} R_{\ldots}^{k+1}\right\} .
\end{aligned}
$$

A simple analysis shows that this theory is superrenormalizable, but the massive ghost is still here. For the case of real poles one can prove that the spin-two part of the propagator has the structure

$$
G_{2}(k)=\frac{A_{0}}{k^{2}}+\frac{A_{1}}{k^{2}+m_{1}^{2}}+\frac{A_{2}}{k^{2}+m_{2}^{2}}+\cdots+\frac{A_{N+1}}{k^{2}+m_{N+1}^{2}},
$$

where for any sequence $0<m_{1}^{2}<m_{2}^{2}<m_{3}^{2}<\cdots<m_{N+1}^{2}$, the signs of the corresponding terms alternate, $A_{j} \cdot A_{j+1}<0$. Therefore, the situation when the ghost is shifted to an infinite energy level is ruled out. 
In order to understand the renormalizability properties of the theory one has to consider the power counting. It is sufficient to consider only vertices $K_{\nu}=2 k+4$, which produce strongest divergences. Then we have $r_{l}=K_{\nu}=2 k+4$ and one can easily arrive at the estimate

$$
D+d=4+k(1-p)
$$

For $k=0$ we meet the standard result, $d \equiv 4$ for $D=0$. Starting from $k=1$ we have superrenormalizable theory, where the divergences exist only for $p=1,2,3$. For $k \geq 3$ we have superrenormalizable theory, where the divergences exist only at the one-loop level, when $p=1$.

So, if we solve the ghost problem someday, there will be many versions of renormalizable and superrenormalizable higher derivative QG theories. In particular, superrenormalizable QG theory described above has some interesting features. For example, in the basic fourth-derivative version, the quantum corrections have an ambiguity related to the choice of gauge-fixing condition. As a consequence of this, there are no well-defined $\beta$ - function for the Newton constant $G$ and for the cosmological constant $\Lambda$. On the contrary, in the superrenormalizable versions there is no such problem, in fact all $\beta$ - functions are well-defined and gauge-fixing independent. Moreover, for $k \geq 3$ it is possible (albeit very difficult) to derive exact $\beta$-functions. Finally, one can say that there are quite a lot of superrenormalizable QG theories with many free parameters, and not a single experiment to fix their values. Perhaps, the main problem of QG is not just a theory, but the lack of experimental data.

\section{What is the problem with ghosts?}

A consistent theory of quantum matter fields on classical curved background can be achieved only if we include the higher derivative terms (6) into the classical action of vacuum. The same action (4) represents also a basis for renormalizable QG theory. What is a problem with massive ghosts which make the higher derivative theories so problematic?

In short, the presence of ghosts created the following problem: the vacuum state of the theory becomes unstable and theory gets inconsistent. This means, for example, that the vacuum is not protected against a spontaneous creation of massive ghost and of a certain amount of normal particles, which compensate the negative energy of the ghost. Other forms of formulating the same problem can be formulated as follows:

In classical systems higher derivatives generate exploding instabilities at the non-linear level, as was discussed originally by Ostrogradsky in 1850 in Ref. 18 and in relation to the gravitational case by Woodard in Ref. 10. One can check that at the linear level the theory is stable, therefore the problem is related to the non-linear level of consideration.

Interaction between ghost and gravitons may violate energy conservation in the massless sector. This possibility has been explored by Veltman in $1963{ }^{\frac{19}{19}}$ In 
short, this means that the quantum scattering of ghost and normal (positive-energy) particles has the feature that the dominating process is accelerating a ghost, which gains more and more negative energy, while the positive compensating energy goes to the outflux of gravitons, in the present case.

So, the situation is such that the presence of ghost makes the theory ill-defined. Therefore, the question is whether it is possible or not that the Lagrangian of the theory admits the existence of the massive ghost excitation, while there is no such particle in reality. The first observation is that, if we do not include the ghost into the in state, the theory will be inconsistent, because interactions between ghost and gravitons will produce the ghost particles in the out state and the $S$-matrix of the theory will be non-unitary. Indeed, one can think that the scattering problems in QG are not the most important ones, especially at the relatively low energies, which means just much below the Planck scale. Assuming this viewpoint, one may not care about the $S$-matrix, especially because the Planck-mass particle may be nonobservable. However, this would be a wrong conclusion, because if massive ghosts really emerge in the theory, this will result in the Veltman scattering and finally to the huge flux of the energy of gravitons. Since we do not observe the emergence of gravitons with the Planck energy densities in experiments (and, in fact, in the real life which would be seriously affected!), it is natural to conclude that the Nature is organized somehow different, such that we do not have the Planck-scale ghosts interacting to gravitons.

\section{History of the fight: physicists against ghost}

Hopefully we have convinced the reader that the situation is somehow contradictory. From one side, we need higher derivatives to construct renormalizable theory of matter fields and quantum theory of the metric itself. Up to some extent the first part is even more relevant, because one can deal with the quantization of the metric in different ways, e.g., switch to the string theory. However, in the case of semiclassical theory we have to deal with a real physics, that means to put quantum fields on curved background. And this really requires the terms (6) to be present in the action. On the other hand, such important achievement as Hawking radiation is, 20 in fact, related to the conformal anomaly, ${ }^{21}$ which results from the renormalization of the terms (6). The same is true for another very important application of quantum theory. The complete version of the Starobinsky model of inflation ${ }^{22}$ is also based on the conformal anomaly ${ }^{23}$ In both cases one can perform the analysis on the basis of the anomaly-induced effective action of gravity, ${ }^{24}$ which is just a natural quantum extension of (6). So, the higher derivatives are necessary and important, hence we can not (and, in some sense, do not like to) get rid of them.

On the other hand, in the presence of higher derivatives one meets massive ghosts, vacuum instability and related difficulties with consistent formulation of the theory at both classical and quantum levels. Hence, it is not a surprise that there were many attempts to find a solution of the ghost problem. Let us briefly discuss 
some of them.

One can construct higher derivative theories of gravity without ghosts $25 \mid 26$ (see also recent works in Ref. 27). All these theories are very similar to the GaussBonnet action, which is free of ghosts because the propagator of the gravitational field behaves in the UV like $1 / k^{2}$. As it was discussed in Ref. 28, this rules out renormalizability in the QG theory. The situation is even more clear in the semiclassical case, when the terms of the action (6) are generated by the matter loops. Obviously, any modification of the gravity action which rules out these terms does not help to make the theory consistent.

The mainstream approach for the ghost problem has been developed in Refs. 29, 30. 31. The idea is to assume the resummation of the perturbative series such that, taking the full propagator instead of the tree-level one, massive ghost becomes unstable and disappears in the out state. Then one can start with the in-state without ghosts and still have an unitary $S$-matrix of the gravitational perturbations. From the technical side, the best realization of this idea requires that the loop corrections shift the position of the ghost pole to the complex plane and that this position becomes gauge-fixing dependent ${ }^{31}$ With these assumptions one can prove the unitarity of the theory. Unfortunately, the existing perturbative and non-perturbative (e.g., 1/N expansion, lattice formulations etc) methods are not sufficient to claim whether these two conditions or at least part of them are satisfied or not 32

An alternative idea has been suggested by Hawking et al ${ }^{33}$ It is necessary to remember that the ghost does not emerge in the theory as independent particle, but only comes together with graviton. There is a chance that the quantum field theory which takes this aspect into account, will be free of instabilities. The realization of this interesting idea requires qualitatively new formulation of quantum field theory. Unfortunately, until now there is no consistent formulation of this sort and hence one can not be sure whether this approach really works or not.

Another possibility is to consider the situation when the ghosts are present,

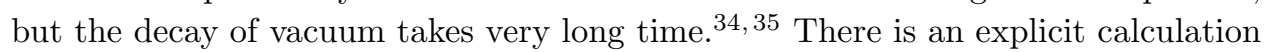
supporting this possibility, $\stackrel{36}{b}$ but it works only for a very small mass of the ghost. On the other hand, in case of higher derivative quantum gravity the typical mass of the ghost is very large, of the Planck order of magnitude.

Finally, there was a very interesting proposal to generalize (18) to the nonpolynomial in $\square$ structure ${ }^{37}$ One can choose the function of $\square$ in such a way that there would be no ghosts. It is supposed that this theory will be superrenormalizable, exactly as (18) is for the polynomial of high (above three) order function. Recently there was a significant activity in exploring this kind of theories at the classical level (see Ref. 38 for the review and further references). At the quantum level, the main questions are how to perform quantization in the non-polynomial theory, and how to perform practical calculations. Another difficult problem is how to evaluate the superficial degree of divergence in such a theory. Gravity has a non-polynomical interactions, and according to the general formula (13) we meet an indefinite output $D+d \sim \infty-\infty$. Then it is difficult to arrive at some definite conclusions about 
whether the theory under discussion is superrenormalizable, renormalizable or even non-renormalizable. Some relevant considerations about this subject can be found in Ref. 39.

We can conclude that the situation with Planck-scale massive ghosts is unclear, in the sense there are several interesting proposals, but no certainty that al least one of them can be successful. In what follows we describe a new approach which is much simpler and is probably working. The main point is the possibility of that the ghosts may be actually not generated from vacuum by interaction with gravitons, if these gravitons do not have energies comparable to the Planck scale. One can note that the spontaneous creation of ghost and gravitons means that the energy density of these gravitons in the given space-time point is of the Planck order of magnitude.

The low-energy classical solutions of the theory (4) with higher derivative terms (6) should be very close to the ones of GR, because the effect of the terms (흠 is going to be Planck-suppressed. Then the theory is, in general, safe from the dangerous ghost instabilities if these classical solutions are stable against small perturbations of the metric. The stability of the theory in the Lyapunov sense (see, e.g., Ref. 40) means that the non-trivial background takes care about all non-linear effects, so if the theory is stable with respect to the small perturbations at the linear level, then the non-linear stability is guaranteed. On the other side, it is known that the spontaneous creation of ghost does not occurs within the linearized theory in flat space-time background (see, e.g., Ref. 10). Therefore, all the question is whether the background metric can change this situation and, if this is the case, what are the conditions for the creation of ghost and/or instabilities.

\section{Stability of classical solutions at low energies}

In our opinion, the most risky assumption which is usually done to rule out the higher derivative theory is that the Ostrogradsky instabilities or Veltman scattering are relevant independent on the energy scale. There is a relatively simple way to check this assumption. Let us take a higher derivative theory of gravity and verify the stability with respect to the linear perturbations on some, physically interesting, dynamical background. If the mentioned assumption is correct, we will observe rapidly growing modes even for the low-energy background and for the low initial frequencies of the gravitational perturbation. On the contrary, if there are no growing modes at the linear level, there will not be such modes even at higher orders. One has to remember that the ghost issue is essentially a tree-level problem, so the study of classical solution is sufficient to draw conclusions about the general situation.

Up to the present moment, the program formulated above has been realized in the following three cases:

1) Cosmological background. In the particular case of de Sitter metric the result is partially known for more than thirty years ${ }^{44}$ and has been repeatedly confirmed ${ }^{43}[45]$ In these papers the theory with semiclassical corrections to the classical 
action (6) has been used. On the other hand, recently the same investigation has been repeated for other cosmological metrics, such as radiation- and dust-dominated Universes ${ }^{7}$ At the same time, in all these papers the relation between instabilities and higher derivative ghosts was never traced back explicitly. This last part has been explored in Ref. 8 and in what follows we shall review the main results of this work.

2) Black hole background. In this case there are conflicting data in the literature, namely the statements about stability 41 and instability ${ }^{42}$ of this solution. The analysis of this case is technically very complicated and we will not discuss it here in details. Let us only mention that it is not clear, to which extent the results depend on the choice of the boundary conditions, on the frequency of initial seeds of perturbations and also on some technical assumptions done in these works.

3) General curved background which is close to a flat space-time. Since the nonlinearities of the perturbations can be taken into account by means of a non-trivial metric background, it looks natural to consider a weak (albeit arbitrary) gravitational field. Such consideration is, in principle, possible ${ }^{8}$ Using normal coordinates and local momentum representation, we have constructed the relevant equations in the lowest non-trivial approximation. It is natural to expect that there will be a smooth transition to the precisely flat case, where ghost does not lead to instability. Probably this, technically complicated, study will end with the conclusion that there are no instabilities if the curvature is much smaller than the square of the Planck mass, which is the unique dimensional parameter in the theory (4). However, the results are not conclusive yet in this part.

Taking the present-day state of art into account, in what follows we shall describe only the situation with the cosmological background, where the results are qualitatively clear and understandable.

\section{Background cosmological solutions}

In principle, one can explore the stability of the classical solution in the theory (4), but for the sake of generality we can include also the semiclassical corrections coming from the massless fields. It is supposed that the effects of massive fields are negligible at the sufficiently low energies.

In the case of massless conformal fields one can set to zero the coefficient of the $R^{2}$-term, $a_{4}=0$, in the action (6), without violating renormalizability. Therefore, the theory of our interest is described by the sum of a classical action (4) with 
$a_{4}=0$ and with the additional anomaly-induced quantum contribution,

$$
\begin{aligned}
\Gamma_{i n d} & =S_{c}\left[g_{\mu \nu}\right]-\frac{3 c+2 b}{36(4 \pi)^{2}} \int_{x} R^{2}(x)+\frac{\omega}{4} \iint_{x} C^{2}(x) G(x, y)\left(E-\frac{2}{3} \square R\right)_{y} \\
& +\frac{b}{8} \iint_{x}\left(E-\frac{2}{3} \square R\right)_{x} G(x, y)\left(E-\frac{2}{3} \square R\right)_{y},
\end{aligned}
$$

where we used compact notations

$$
\int_{x}=\int d^{4} x \sqrt{-g}, \quad \text { and } \quad \Delta_{4} G(x, y)=\delta(x, y) .
$$

Furthermore,

$$
\Delta=\square^{2}+2 R^{\mu \nu} \nabla_{\mu} \nabla_{\nu}-\frac{2}{3} R \square+\frac{1}{3}\left(\nabla^{\mu} R\right) \nabla_{\mu}
$$

is the conformal self-adjoint Paneitz operator, coefficients $\omega, b, c$ depend on the number of quantum fields and $S_{c}\left[g_{\mu \nu}\right]$ is an arbitrary conformal invariant functional of the metric. Further details about derivation of (21) can be found, e.g., in Ref. 45 ,

In order to understand the effect of quantum terms on the conformal factor of the metric, let us consider the equation for this factor $a(t)$, Consider unstable inflation, matter (or radiation) dominated Universe and assume that the Universe is close to the classical FRW solution. The equation is

$$
\begin{array}{r}
\frac{\ddot{a}}{a}+\frac{3 \dot{a} \dot{a}}{a^{2}}+\frac{\ddot{a}^{2}}{a^{2}}-\left(5+\frac{4 b}{c}\right) \frac{\ddot{a} \dot{a}^{2}}{a^{3}}-2 k\left(1+\frac{2 b}{c}\right) \frac{\ddot{a}}{a^{3}} \\
-\frac{M_{P}^{2}}{8 \pi c}\left(\frac{\ddot{a}}{a}+\frac{\dot{a}^{2}}{a^{2}}+\frac{k}{a^{2}}-\frac{2 \Lambda}{3}\right)=-\frac{1}{3 c} \rho_{\text {matter }}
\end{array}
$$

where we have also introduced the matter term for illustrative purpose. Also, $k=$ $0, \pm 1$ and $\Lambda$ is the cosmological constant. It is easy to see how the things change in this equation when the time change. First of all, let us consider the empty universe, with $\rho_{\text {matter }} \rightarrow 0$. In this case one can find particular solutions (see Ref. 22 and also Ref. 46 for the case with cosmological constant)

$$
a(t)= \begin{cases}a_{o} \exp (H t), & k=0 \\ a_{o} \cosh (H t), & k=1 \\ a_{o} \sinh (H t), & k=-1\end{cases}
$$

where Hubble parameter takes two constant values

$$
H=H_{ \pm}=\frac{M_{P}}{\sqrt{-32 \pi b}}\left(1 \pm \sqrt{1+\frac{64 \pi b}{3} \frac{\Lambda}{M_{P}^{2}}}\right)^{1 / 2}
$$

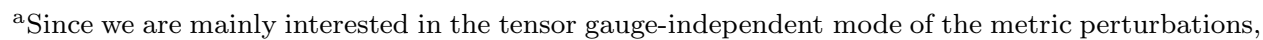
there is no problem to assume that the classical $R^{2}$-term is absent, since this term does not influence too much the dynamics of this mode.
} 
Let us note that the coefficient $b$ is negative for any particle content of the theory contributing to quantum terms. For the small cosmological constant $\Lambda \ll M_{P}^{2}$ the two solutions (26) boil down to

$$
H_{+}=\frac{M_{P}}{\sqrt{-16 \pi b}}, \quad H_{-}=\sqrt{\frac{\Lambda}{3}} .
$$

Obviously, the first solution here is usual Starobinsky inflation (initial part of it, better say) and the second one is the usual dS solution without quantum corrections. What we need here is the stability of the second of these solutions with respect to the tensor perturbations of the metric.

One can first perform a simple test of the model, by considering the stability of the low-energy solution with $H_{-}$with respect to the perturbations of the conformal factor (see second reference in Ref. 46). Consider $H \rightarrow H_{-}+$const $\cdot e^{\lambda t}$ and arrive at

$$
\lambda^{3}+7 H_{0} \lambda^{2}+\left[\frac{(3 c-b) 4 H_{0}{ }^{2}}{c}-\frac{M_{P}^{2}}{8 \pi c}\right] \lambda-\frac{32 \pi b H_{0}{ }^{3}+M_{P}^{2} H_{0}}{2 \pi c}=0 .
$$

The solutions of this equation have positive real parts

$$
\lambda_{1}=-4 H_{0}, \quad \lambda_{2 / 3}=-\frac{3}{2} H_{0} \pm \frac{M_{P}}{\sqrt{8 \pi|c|}} i,
$$

indicating the absence of growing modes. Obviously, the positive cosmological constant $\Lambda>0$ protects the low-energy dS solution from higher-derivative instabilities in this case.

One can regard the two dS solutions (27) as extreme states of the Universe.46] 47 The first of these solutions is the initial phase of the Starobinsky inflation and the last one is the distant future of the Universe when the effect of all kinds of matter becomes irrelevant and only cosmological constant will drive the accelerated expansion. What is important for us is that, in the low-energy regime of a late Universe, the solution with $H_{-}$provides an extremely precise approximation for the solution with quantum terms taken into account. In the absence of quantum term (21) this is an exact solution, because Eq. (6) with $a_{4}=0$ does not affect the dynamics of the conformal factor. But even if the quantum term (24) is taken into account, it is still a perfect approximation. The reason that the theory without matter has only two dimensional parameters, $M_{P}$ and $\Lambda$. Any correction to $H_{-}$is given by a positive power of the ratio $\Lambda / M_{P}^{2}$, which is of the order of $10^{-120}$. So, we can safely use this background solution at low energies.

Let us now consider the case with the nontrivial matter contents, $\rho_{\text {matter }}$. Consider the late time epoch. It is easy to see that the terms of the first line of (24), which are of the quantum origin, behave like $t^{-4}$. At the same time the second-line terms, of the classical origin, all behave like $t^{-2}$ (see Ref. 48 for more detailed discussion). Obviously, the quality of the classical approximation for the solution $a(t)$ becomes better for $t \rightarrow \infty$ and can be considered a very good one in the late epoch of the Universe. 


\section{Gravitational waves and ghosts}

Now we are in a position to explore the dynamics of the gravitational waves on the background of cosmological solutions described in the previous section. For this end we consider small perturbation

$$
g_{\mu \nu} \rightarrow g_{\mu \nu}+h_{\mu \nu}, \quad h_{0 \mu}=0, \quad \partial_{i} h^{i j}=0 \quad \text { and } \quad h_{i i}=0,
$$

where the last three conditions mean synchronous coordinate condition and fixing the gauge freedom such that we deal with the tensor mode only. The background metric should be $g_{\mu \nu}^{0}=\left\{1,-\delta_{i j} a^{2}(t)\right\}$, where $a(t)$ can be chosen as cosmological constant-, radiation- or dust-dominated classical solution. Finally, our notations are $\mu=0, i$ and $i=1,2,3$. In order to explore the time dynamics of the gravitational waves one can make a partial Fourier transformation

$$
h_{\mu \nu}(t, \vec{r})=\int \frac{d^{3} k}{(2 \pi)^{3}} e^{i \vec{r} \cdot \vec{k}} h_{\mu \nu}(t, \vec{k})
$$

and assume that the modes with different momenta do not interact between each other. Then $k=|\vec{k}|$ becomes a constant parameter and one can deal with an ordinary differential equation instead of a partial one.

In the original papers we worked with both classical case described only by the action (4) in Ref. 8 and with the theory which includes semiclassical corrections (21) in Ref. 7. It was shown that the effect of these semiclassical corrections is negligible when we deal with the sufficiently small perturbations and sufficiently weak background. The qualitative explanation of this fact is that all the terms in (21) are at least of the third order in curvature tensor, or reduce to the less relevant $R^{2}$-term. Therefore, for the reason of compactness we will restrict ourselves by the purely classical case and also keep the cosmological constant zero and the space section of the space-time manifold plane. Then the equation for the perturbations have the form

$$
\begin{aligned}
& \frac{1}{3} \dddot{h}+2 H \dddot{h}+\left(H^{2}+\frac{M_{P}^{2}}{32 \pi a_{1}}\right) \ddot{h}+\frac{2}{3}\left(\frac{1}{4} \frac{\nabla^{4} h}{a^{4}}-\frac{\nabla^{2} \ddot{h}}{a^{2}}-H \frac{\nabla^{2} \dot{h}}{a^{2}}\right) \\
- & {\left[H \dot{H}+\ddot{H}+6 H^{3}-\frac{3 M_{P}^{2} H}{32 \pi a_{1}}\right] \dot{h}-\left[\frac{M_{P}^{2}}{32 \pi a_{1}}-\frac{4}{3}\left(\dot{H}+2 H^{2}\right)\right] \frac{\nabla^{2} h}{a^{2}} } \\
- & {\left[\left(24 \dot{H} H^{2}+12 \dot{H}^{2}+16 H \ddot{H}+\frac{8}{3} \dddot{H}\right)-\frac{M_{P}{ }^{2}}{16 \pi a_{1}}\left(2 \dot{H}+3 H^{2}\right)\right] h=0 . }
\end{aligned}
$$

Already at this level one can see that the equation depends only on the coefficient of the Weyl-squared term $a_{1}$ in the action (6) and not on other terms, as one should expect.

The analysis of the equation (32) and its semiclassical generalization has been done in Ref. 8 and Ref. 7, correspondingly. Let us present here only qualitative results, which were achieved by both analytical and numerical methods. The analytical method was based on the following idea. One can approximately treat all coefficients as constants, assuming that the time variation of the Hubble parameter 
and its derivatives performs slower that the one of the perturbations. In this case the consideration can be performed by conventional elementary methods. The numerical methods included the CMBEasy software or Wolfram's Mathematica, and provided the results which were perfectly consistent with the mentioned analytic approach.

The net result is that the stability is completely defined by the sign of the coefficient $a_{1}$ of the Weyl-squared term $\mathrm{b}$. The most relevant observation is that the sign of this term defines whether graviton or ghost has positive or negative kinetic energy!

One can distinguish the following two cases:

- The coefficient of the Weyl-squared term is negative, $a_{1}<0$. Then

$$
G_{\text {spin }-2}(k) \sim \frac{1}{m^{2}}\left(\frac{1}{k^{2}}-\frac{1}{k^{2}+m^{2}}\right), \quad m \propto M_{P} .
$$

In this case there are no growing modes up to the Planck scale, $\vec{k}^{2} \approx M_{P}^{2}$. For the dS background this is in a perfect agreement with the previous results of Ref. 44 and Ref. 43. It is remarkable that when the frequency $k=|\vec{k}|$ is getting close to the Planck scale, the growing modes start to show up. From the physical side this means that the higher derivative theory (4) is actually stable against ghost-induced perturbations, but only for the frequencies below the Planck cut-off. Some plots illustrating this situation are shown in Figure 2

- The classical coefficient of the Weyl-squared term is positive, $a_{1}>0$. In this case the propagator of the tensor mode has the form

$$
G_{\text {spin }-2}(k) \sim \frac{1}{m^{2}}\left(-\frac{1}{k^{2}}+\frac{1}{k^{2}+m^{2}}\right), \quad m \propto M_{P} .
$$

With this "wrong" sign of $a_{1}$, the massless graviton is becoming a ghost. On the contrary, massive spin-2 particle in this case has positive energy. As one could expect, in this case there is no Planck-mass threshold and, as we have found, there are rapidly growing modes at any scale of frequencies. This example is artificial, but very illustrative, for it explicitly shows the relation between mass of the ghosts and the stability of classical solutions.

Coming back to the physical case $a_{1}<0$, the natural interpretation of the result is that, at low energies, the massive ghosts are present only in the vacuum state. There are no even one of such excitations "alive" until the typical energy scale remains below the Planck mass threshold. As far as the frequency comes close to $M_{P}$, the ghosts start to be generated from vacuum and we observe strong instabilities.

One more verification of the correspondence between ghosts and stability with respect to tensor modes can be obtained for the superrenormalizable theory (18).

\footnotetext{
${ }^{\mathrm{b}}$ Let us mention that the same is true in the semiclassical case with the non-zero coefficient $c$, which corresponds to the classical $a_{4}$.
} 


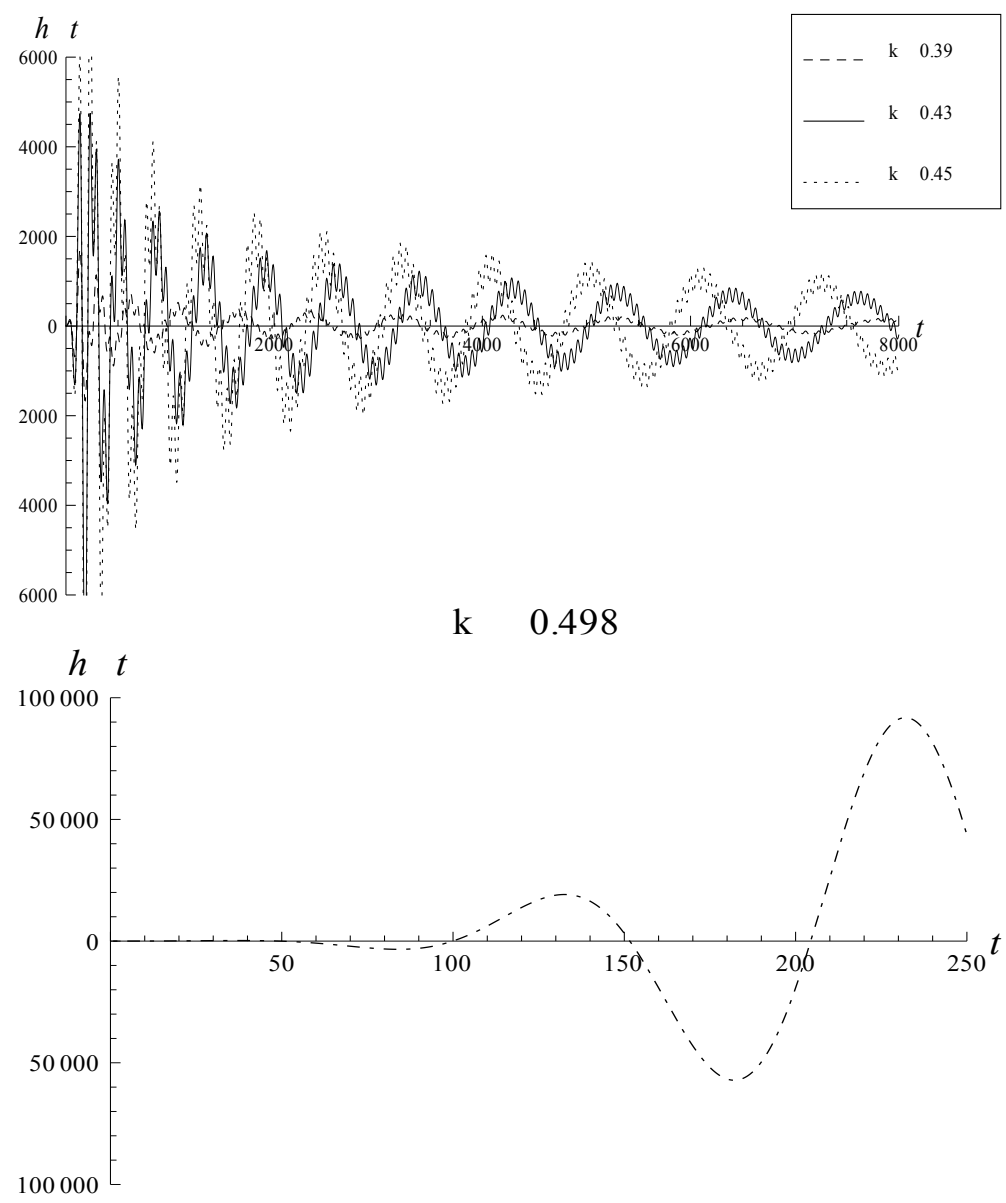

Fig. 2. Illustrative plots for the case of radiation-dominated Universe. There are no growing modes up to the frequency $k \approx 0.5$ in Planck units. Starting from this value, one can observe the massive ghost making destructive work.

According to the result (19), if we include just one dAlembert operator $\square$, there will be only one extra massive tensor particle and it is not a ghost, hence the stability conditions should not be modified. And this is exactly what we have observed in this case by means of numerical and analytical methods, in Ref. 8, In particular, the threshold frequency almost does not change if the mass of the ghost is the same. Once again, we observe a correspondence between the presence and mass of the ghosts and (in)stability of the classical solution.

\section{Conclusions and further perspectives}

One should definitely quantize both matter and gravity, for otherwise the theory would not be complete. Indeed, the quantum matter is something essentially more certain than the quantum metric, simply because quantization of matter is really 
experimentally supported, in all senses. Since it is not possible to deal with the quantization of matter fields without higher derivatives terms (6), the main question is not whether we like these terms or not. In our opinion the question is to explain why these terms do not produce destructive instabilities in the classical gravitational solutions.

For QG with higher derivatives, the propagator includes massive nonphysical mode(s) called ghosts. These massive ghosts are capable to produce terrible instabilities, but for some unknown reason our world is stable and it is interesting to understand why this happens. There are many ways to deal with this fact, and here we reviewed some of them. It turns out that, at least in the cosmological case, ghosts are not produced at the energy scales far below the Planck mass. If there is no at least one such ghost excitation in the initial spectrum, there are no instabilities at the linear approximation and the Lyapunov theorems guarantee that this will be the case, also, at the non-linear level.

It is possible that massive ghosts do not pose real danger below the Planck scale. However, in order to check this fact, it is important to have well-established results for other backgrounds, starting from the Schwarzschild and Kerr solutions. On the other hand, it would be interesting to analyse the general metric case in the approximation of weak curvature tensor components.

Assuming that our conjecture about the situation with ghosts "sleeping" in the vacuum state is correct, the higher derivative gravity becomes a perfect candidate to be an effective QG below the Planck scale. Then we have to answer the question of whether the effect of this theory at low energies is the same of the low-energy quantum GR or not, as it was discussed in Ref. 49.

It is clear that the energy scale below Planck mass covers most of the possible applications. On the other hand, there is a conceptually important question of what happens with the ghosts above $M_{P}$. In this case we need some new ideas. The solution can be related to string theory, or to some new principles of Physics which we do not know yet. In principle, on of the options would be some principle which forbids the Planck densities of energy to form. For instance, some hypothesis which closely fit this requirements, can be found in the recent works, $\frac{50}{b u t}$, in general, this problem remains open.

\section{Acknowledgments}

This short review is based on original papers by the authors and their collaborators. We are grateful to them, especially to Julio Fabris, for the contributions to these works. Another source of this review are seminars, mainly given by I.Sh. between 2012 and 2014. We appreciate the contribution of those who invited him to speak about the subject and also those who asked questions. The work of the authors was partially supported by CNPq, CAPES, FAPEMIG and (in case of I.Sh.) ICTP. 


\section{References}

1. N.D. Birell and P.C.W. Davies, Quantum Fields in Curved Space (Cambridge University Press, Cambridge, 1982).

2. A.A. Grib, S.G. Mamaev and V.M. Mostepanenko, Vaccum Quantum Effects in Strong Fields. (Moscow, Atomizdat. In Russian, 1980); English translation: St. Petersburg, Friedmann Laboratory Publishing, 1994.

3. I.L. Buchbinder, S.D. Odintsov and I.L. Shapiro, Effective Action in Quantum Gravity (IOP Publishing, Bristol, 1992).

4. L.E. Parker and D.J. Toms, Quantum Field Theory in Curved Spacetime: Quantized Fields and Gravity (Cambridge University Press, 2009).

5. B. Zwiebach, Phys. Lett. B 156315 (1985).

6. A.L. Maroto and I.L. Shapiro, Phys. Lett. B 41434 (1997).

7. J.C. Fabris, A.M. Pelinson, F. de O. Salles and I.L. Shapiro, JCAP 02019 (2012); arXiv: 1112.5202 .

8. F. de O. Salles and I.L. Shapiro, Phys. Rev. D 89084054 (2014); arXiv:1401.4583.

9. P.M. Lavrov and I.L. Shapiro, Phys. Rev. D 81044026 (2010); arXiv:0911.4579

10. R. Woodard, Lect. Notes Phys. 720403 (2007).

11. S. Weinberg, Ultraviolet divergences in quantum theories of gravitation. In "General Relativity: An Einstein centenary survey", ed. S. W. Hawking and W. Israel. (Cambridge University Press. pp. 790831, 1979).

12. M. Niedermaier and M. Reuter, Living Rev. Rel. 95 (2006);

R. Percacci, "Asymptotic Safety", in D. Oriti, Approaches to Quantum Gravity: Towards a New Understanding of Space, Time and Matter, (Cambridge University Press, 2009); arXiv:0709.3851.

13. K.S. Stelle, Phys. Rev. D 16953 (1977).

14. G. 't Hooft and M.J.G. Veltman, Ann. Poincare Phys. Theor. A 2069 (1974).

15. S. Deser and P. van Nieuwenhuizen, Phys. Rev. D 10401 (1974); Phys. Rev. D 10411 (1974); S. Deser, H.Sh. Tsao and P. van Nieuwenhuizen, Phys. Rev. D 103337 (1974).

16. M.H. Goroff and A. Sagnotti, Nucl. Phys. B 266709 (1986).

17. M. Asorey, J.L. López and I.L. Shapiro, Int. Journ. Mod. Phys. A 125711 (1997).

18. M. Ostrogradski, Mem. Ac. St. Petersbourg VI 4385 (1985).

19. M.J.G. Veltman, Physica 29186 (1963).

20. S. W. Hawking, Nature (London) 24830 (1974); Commun. Math. Phys. 43199 (1975), Erratum-ibid. 46206 (1976).

21. S.M. Christensen and S.A. Fulling, Phys. Rev. D 152088 (1977).

22. A.A. Starobinski, Phys.Lett. B 9199 (1980).

23. M.V. Fischetti, J.B. Hartle and B.L. Hu, Phys. Rev. D 201757 (1979).

24. R.J. Riegert, Phys. Lett. B 13456 (1980); E.S. Fradkin and A.A. Tseytlin, Phys. Lett. B 134187 (1980).

25. D.E. Nevill, Phys. Rev. D 183535 (1978); Phys. Rev. D 21867 (1980); Phys. Rev. D 231244 (1981); 25573 (1982).

26. E. Sezgin and P. van Nieuwenhuizen, Phys. Rev. D 213269 (1981); E. Sezgin, Phys. Rev. D 241677 (1981).

27. J.A. Helayel-Neto, C.A. Hernaski, B. Pereira-Dias, A.A. Vargas-Paredes, V.J. Vasquez-Otoya, Phys. Rev. D 82064014 (2010), arXiv:1005.3831.

28. I.L. Shapiro, Phys. Repts. 357113 (2002).

29. E. Tomboulis, Phys. Lett. B 70361 (1977); Phys. Lett. B 9777 (1980); Phys. Rev. Lett. 521173 (1984).

30. A. Salam and J. Strathdee, Phys. Rev. D 184480 (1978).

31. I. Antoniadis and E.T. Tomboulis, Phys. Rev. D 332756 (1986). 
32. D.A. Johnston, Nucl. Phys. B 297721 (1988).

33. S.W. Hawking, In Quantum Field Theory and Quantum Statistics 2. 129 (1985); S.W. Hawking and Th. Hertog, Phys. Rev. D 65103515 (2002).

34. I. Antoniadis, E. Dudas and D.M. Ghilencea, Nucl.Phys. B 76729 (2007), hep-th/0608094

35. J. Garriga and A. Vilenkin, JCAP 1301036 (2013), arXiv: 1202.1239.

36. M. Jaccard, M. Maggiore and E. Mitsou, Phys. Rev. D 88044033 (2013), arXiv: 1305.3034

37. E.T. Tomboulis, Superrenormalizable gauge and gravitational theories, hep-th/9702146

38. L. Modesto, Astron. Rev. 8.24 (2013), arXiv:1202.3151

F. Briscese, L. Modesto and S. Tsujikawa, Super-renormalizable or finite completion of the Starobinsky theory, arXiv:1308.1413 [hep-th].

L. Modesto and S. Tsujikawa, Phys. Lett. B $\mathbf{7 2 7} 48$ (2013), arXiv:1307.6968.

39. L. Modesto, Phys. Rev. D 86044005 (2012), arXiv:1107.2403,

L. Modestoa and L. Rachwal, Super-renormalizable $\&$ Finite Gravitational Theories. arXiv:1407.8036.

40. L. Cesari, Asymptotic Behavior and Stability Problems in Ordinary Differential Equations, (Springer; 3rd Edition, 2012).

41. B. Whitt, Phys. Rev. D 32379 (1985).

42. Yu.S. Myung, Phys. Rev. D 88024039 (2013), arXiv:1306.3725.

43. S.W. Hawking, T. Hertog and H.S. Reall, Phys. Rev. D 63083504 (2001), hep-th/0010232

44. A.A. Starobinsky, JETP Lett. 30682 (1979) [Pisma Zh. Eksp. Teor. Fiz. 30719 (1979)]; Zh. Eksp. Teor. Fiz. 34460 (1981); Sov. Astron. Lett. 9302 (1983).

45. J. C. Fabris, A. M. Pelinson and I. L. Shapiro, Nucl. Phys. B 597539 (2001); [Erratumibid. 602644 (2001)], hep-th/0009197.

46. A.M. Pelinson, I.L. Shapiro and F.I. Takakura, Nucl. Phys. B 648417 (2003); Nucl. Phys. (Proc. Suppl.) B 127182 (2004).

47. I.L. Shapiro, Int. Journ. Mod. Phys. D 111159 (2002);

I.L. Shapiro, J. Solà, Phys. Lett. B 53010 (2002).

48. A.M. Pelinson and I.L. Shapiro, Phys. Lett. B 694467 (2011), arXiv: 1005.1313; Intern. Journ. Mod. Phys. A 263759 (2011).

49. I.L. Shapiro, Int. J. Mod. Phys. A 241557 (2009), arXiv:0812.3521

50. G. Dvali, S. Folkerts, and C. Germani, Phys. Rev. D 84024039 (2011), arXiv:1006.0984

G. Dvali and C. Gomez, Fortschr. Phys. 63742 (2013), arXiv:1112.3359 


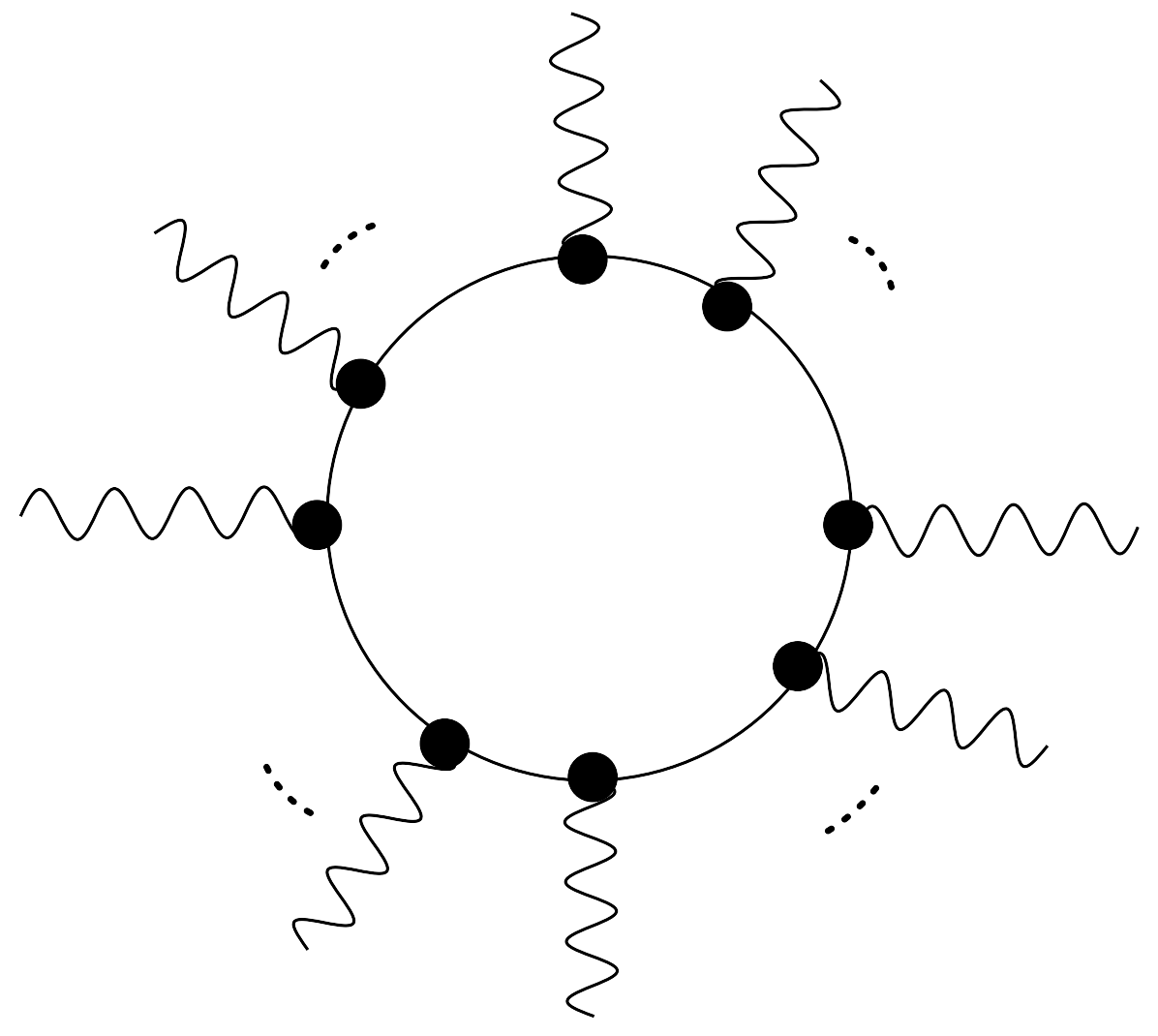

